\title{
Push-Pull Two-layer Super-Peer based P2P Live Media Streaming
}

\author{
POO KUAN HOONG, HIROSHI MATSUO \\ Department of Computer Science \& Engineering \\ Nagoya Institute of Technology \\ Showa, Gokiso, Nagoya, 466-8555, JAPAN \\ khpoo@matlab.nitech.ac.jp, matsuo@nitech.ac.jp
}

\begin{abstract}
Peer-to-peer (P2P) file sharing has become increasingly popular, accounting for as much as $70 \%$ of Internet traffic by some estimates. Recently, we have been witnessing the emergence of a new class of popular P2P applications, namely, P2P audio and video streaming. In this paper, we propose and investigate a full distributed, scalable, and cooperative protocol for live video streaming in an overlay peer-to-peer network. Our protocol, termed P2P Super-Peer based Unstructured Live Media Streaming (PALMS-SP), makes use of combination of push-pull scheduling methods to achieve high performance (in term of delay, stream continuity, cooperation, etc.). The main contribution of PALMS-SP is that it reduces the end-to-end streaming delay and in turn results better delivered quality. We have extensively evaluated the performance of PALMS-SP. Our experiments demonstrate that PALMS-SP with the existence of super-peers achieves better streaming quality in comparison with other existing streaming applications.
\end{abstract}

Key Words: - peer-to-peer, streaming, overlay, push-pull, super-peers

\section{INTRODUCTION}

Peer-to-peer (P2P) file sharing has become increasingly popular, accounting for as much as $70 \%$ of Internet traffic by some estimates. Recently, we have been witnessing the emergence of a new class of popular P2P applications, namely, P2P audio and video streaming. While traditional $\mathrm{P} 2 \mathrm{P}$ file distribution applications target elastic data transfers, P2P streaming focuses on the efficient delivery of audio and video content under tight timing requirements. Still in its infancy, both live and on-demand P2P streaming have the potential of changing the way we watch TV, providing ubiquitous access to a vast number of channels, personalizing your TV experience, and enabling roaming TV services. For a long time, traditional approaches that are client/server based e.g., Akamai (Akamai) have been used for streaming multimedia applications over the Internet.
Over the past few years, P2P networks have emerged as a promising approach for distribution of multimedia content over a network. Some P2P network related researches are by the following authors (Guo et al., 2003), (Hefeeda et al., 2003), (Padmanabhan et al., 2003), (Ye et al., 2004), (Zhang et al., 2005). One form of P2P network, the peer-to-peer overlay, offers a promising approach to support one-to-many multimedia streaming applications without any special support from the network, called P2P streaming. The basic building blocks for P2P streaming, called nodes or peers, are no longer passive receivers of data but instead can act both as clients and servers at the same time. Stream data are simultaneously received, played, and passed to other connected peers. The goal of $\mathrm{P} 2 \mathrm{P}$ streaming mechanisms is to maximize delivered quality to individual peers in a scalable fashion despite the heterogeneity and asymmetry of their access link bandwidth. An effective P2P streaming mechanism depends on the effective utilization of the outgoing bandwidth of most participating peers.

\footnotetext{
Corresponding Author : Poo Kuan Hoong, Department of Computer Science \& Engineering, Nagoya Institute of Technology, Showa, Gokiso, Nagoya, 466-8555, JAPAN

Tel : 0081-90-4440-9062 Fax : 0081-52-744-1355
} 
In live P2P streaming, the media stream is a continuous flow of media data encoded from the streaming server. Media content generated must be delivered to participating nodes under a tight time constraint. Nodes should be able to receive media content before the playout deadline or the media content will be considered obsolete and discarded.

In this paper, we propose and study the selforganizing, decentralized protocol capable of building and maintaining two-layer super-peer based, overlay topologies for P2P streaming live and non-interactive media streaming, called PALMS-SP (P2P Super-Peer based Unstructured Live Media Streaming). Similar to DONet (Zhang et al., 2005), PALMS-SP is based on data-driven and receiver-based that is built on a super-peer based two-layer unstructured overlay media streaming. PALMS-SP is designed to operate in conditions where nodes have heterogeneous bandwidths and resources. PALMS-SP defines the two layers network to simplify the complexity of streaming services. The existence of superpeers makes the network to be more effective because they combine the efficiency of the centralized client-server model with the autonomy, load balancing, and robustness of distributed search. They also take advantage of the heterogeneity of capabilities across peers. Generally, super-peers are nodes that are faster and/or more reliable than "ordinary" nodes that take on server-like responsibilities and provide services to a set of clients. Super-peers allow decentralized networks to run more efficiently by exploiting heterogeneity and distributing load to machines that can handle the burden. On the other hand, this architecture does not inherit the flaws of the client/server model, as it allows multiple, separate points of failure, increasing the health of the P2P network. In comparison to DONet, which only employs pure pull method for packet scheduling, PALMS-SP employs a combination of two methods for media streaming, namely the pull method and push method.

\section{OVERVIEW OF PALMS-SP}

PALMS-SP is based on data-driven and receiver-based unstructured two-layer superpeer based overlay media streaming. It is designed to operate in scenarios where the nodes have heterogeneous and variable bandwidth resources. For the ease of exposition, we refer to the media source as the streaming server and receivers as ordinary peer. The term peers and nodes are interchangeable, and refer to the all the ordinary peers. We consider a network consisting of a large collection of nodes. The network is highly dynamic; new nodes may join at any time, and existing nodes may leave, either voluntarily or by crashing.

PALMS-SP consists of three major components: (i) overlay construction mechanism, which organizes participating peers into a two-layer super-peer based overlay; (ii) streaming scheduling mechanism, which determines the delivery of content from the streaming source to individual nodes through the overlay; and (iii) super-peer management mechanism, which determines which nodes may switch role at will from a ordinary peer to super-peer status. In the following subsections, we describe these components in PALMS-SP.

\section{OVERLAY CONSTRUCTION MECHANISM}

In PALMS-SP, nodes are functionally identical. They are free to exchange control information and media content data from the stream. Each peer maintains a certain number of connected nodes that are known as neighbors. Each node can potentially communicate with every other node in the network. Each node receives media content from a certain number of neighbor nodes and relays the content to a certain number of neighbor nodes. Nodes are heterogeneous: they differ in their computational, storage capabilities, and bandwidth. Nodes may act as super-peers or ordinary nodes. Each super-peer $S P$ is associated with a capacity value $\max (S P)$ 
that represents the maximum number of ordinary nodes associated to a super-peer $S P$.

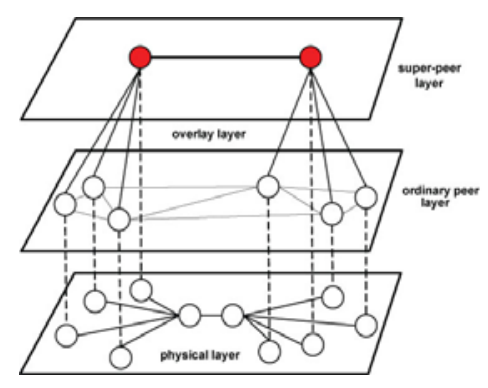

Figure 1 : Two-layer overlay network composed of ordinary peer and super-peer layers

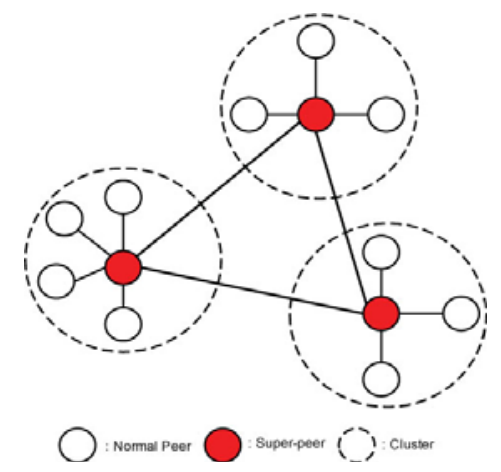

Figure 2: Traditional super-peer network

The basic task of the overlay construction mechanism component for each node is to be in charge of finding appropriate super-peer and neighbors for each node through the gossip method so that the application layer network can be successfully built up. To join the streaming session, a new peer contacts the bootstrapping node, (streaming server in the case of PALMS-SP) to learn about super-peers and other participating peers upon arrival. Streaming server is selected as streaming server persists during the lifetime of streaming and its identifier/address is universally known. This could be regarded as the login/bootstrapping process. The bootstrapping node returns a list of selected super-peers that can potentially serve as parent nodes. The new peer contacts these potential super-peers to determine whether they are able to accommodate a new child node. This is by determining whether the super-peer still has enough allocation slots on the outgoing degree. In the case of PALMS-SP, each peer is associated to exactly one and only super-peer. The number of child nodes associated to a super-peer is pre-determined. As shown in Figure 1, an overlay network consists of two layers, namely ordinary peers layer (lower) and super-peer (higher) layers. The ordinary peer and super-peer layers are composed of a set of ordinary peers and a set of super-peers, respectively. A collection of a super-peer $S P$ and its ordinary peers $O P_{1}, O P_{2}, \ldots O P_{n}(n \geq 1)$, and it is referred to as a cluster $C_{S P}$. A superpeer $S P_{i}$ is connected with another super-peer $S P_{j}$ at the super-peer layer.

The PALMS-SP topology can be summarized as the following:

- each node is either super-peer or a normal peer;

- $\quad$ each ordinary peer $O P$ is associated to exactly one super-peer $S P$;

- the number of ordinary nodes associated to a super-peer $S P$ does not exceed $\max (S P)$.

In traditional super-peer networks shown in Figure 2, ordinary peers in a cluster cannot directly communicate with each other. The ordinary peers have to communicate with each other through super-peer in the cluster. It takes at least two hops to delivery message from an ordinary peer to another ordinary peer. In this paper, we assume each ordinary peer can directly communicate with every neighbor peer in a cluster. Because of this assumption, the number of communication between a super-peer and its ordinary peers can be reduced and the super-peer has a lighter workload.

Each node has a member table that contains a list of neighbor nodes obtained from the superpeer. The information in member tables is encapsulated into a UDP packet and exchanged among neighbors periodically. Each node updates its member table in accordance with the member table sent by its neighbors. A superpeer $S P$ holds all the information on service of 
every ordinary peer in a cluster $C_{S P}$. Each node sends a periodical heartbeat message to update its super-peer. If a node does not update its super-peer periodically, it will be removed from the member table. Once a node leaves, superpeer will broadcast a "leave message" to all its ordinary peers within its cluster. The nodes that receive this message will delete the respective node from its member table as well. Therefore, the failure of any neighbors can be detected by constantly monitoring periodical messages from super-peer.

In order to locate a better neighbor, which has higher uplink, a peer in PALMS-SP periodically replaces the neighbor with the least contribution by selecting nodes with higher scores (the ratio of uploaded packets over downloaded packets). This operation helps each node maintain a stable number of partners in the presence of node departures, and it also helps to discourage the existence of free riders within the network.

\section{STREAMING SCHEDULING}

PALMS-SP employs a swarm-like content delivery mechanism that is similar to BitTorrent (B. Cohen, 2003). Nodes in the swarm protocol will be attracted to nodes that possess newly generated content. The main advantages of swarming content delivery is its ability to effectively utilize the outgoing bandwidth of participating peers and its robustness against the dynamics of peers arrival and departure, which is also known as churn.

The streaming scheduling mechanism of each node is responsible for exchanging packets with all its neighbors. Swarm-like content delivery is incorporated in PALMS-SP. Each peer periodically generates a report i.e., buffer map of its newly received packets and sends it to its neighbor nodes. Each peer periodically requests a subset of required packets from each neighbor node based on the reports received. The pull method is deployed to fetch absent packets from its neighbor nodes and in turn tries its best to deliver packets requested by the neighbors. Packets requested by the pull method are determined by the packet scheduling algorithm, which is much similar to the data-driven approach in DONet (Zhang et al., 2005).

Every node maintains a window of interest, which is the set of sequence packets that the node is interested in acquiring at the current time. Figure 3 illustrates the fundamental concept of the sliding window. A sliding window of availability contains the list of segments available for each node. This is the information for the buffer map shared with other neighbor nodes. The node slides its window of interest forward over time as new packets stream in. If a packet has not been received by the time it "falls off" the trailing edge of the window, the node will consider that packet lost or obsolete and will no longer try to acquire it. Figure 4 shows the buffer state of a node at a specific given time.

To accommodate the bandwidth heterogeneity among peers, the content is encoded with Multiple Description Coding (MDC). Generally, MDC organizes the streaming content into several sub-streams where each sub-stream can independently decoded. The use of MDC for video streaming has been widely studied. Padmanabhan et al. propose that introducing redundancy can provide robustness in media streaming (Padmanabhan et al., 2003). The delivered quality to each peer is proportional to the number of independent substreams it receives. With MDC coding, each peer is able to receive the proper number of substreams that are delivered through the combination push-pull streaming mechanism.

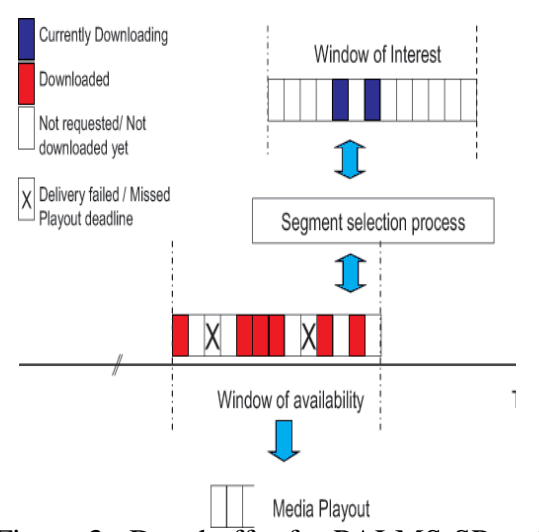

Figure 3 : Data buffer for PALMS-SP node 


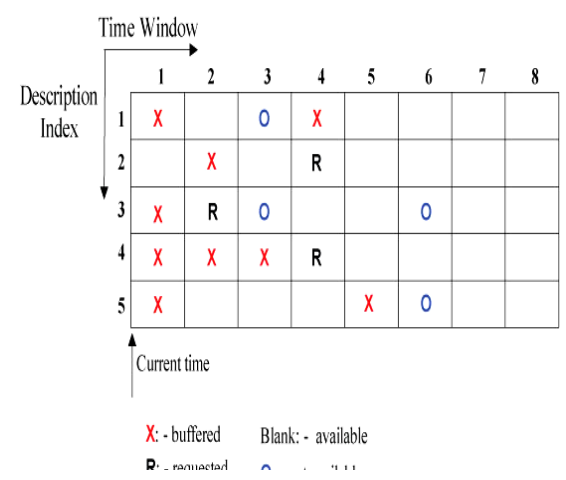

Figure 4 : Buffer state of a node

\section{SUPER-PEER MANAGEMENT MECHANISM}

At the super-peer layer, a super-peer is connected with other super-peers in a flat unstructured overlay network. The ordinary peer and super-peer layers are composed of a set of ordinary peers and a set of super-peers respectively. One of the main obstacles for super-peers network is the super-peer selection. The super-peer selection problem is highly challenging because in the peer-to-peer environment, a large number of super-peers must be selected from a huge and dynamically changing network in which neither the node characteristics nor the network topology is known priori. Thus, simple strategies such as random selection don't work. Super-peer selection is more complex that classic dominating set and p-centers from graph theory, known as the NP-hard problems, because it must respond to the dynamicity of nodes join and leave (churn) and function in an environment that is highly heterogeneous.

The best know example of super-peer selection in a peer-to-peer application is the gnutella (Gnutella, 2003) protocol for selection of ultrapeers - peers with sufficient bandwidth and processing power to serve as proxies for other peers. The use of ultrapeers reduces network traffic and speeds up content delivery. In gnutella, any peer can select itself as an ultrapeer if it meets the following requirements : it has been up for at least 5 minutes, has high bandwidth, sufficient processing power, able to handle a large number of simultaneous TCP connections, and if not behind any firewall or NAT. The ultrapeer selection protocol dynamically adjusts the number of super-peers as follows: if a leaf peer cannot find an ultrapeer with free slots, it can promote itself to be an ultrapeer. Ultrapeers also can downgrade themselves when they are no longer serving as any leaf nodes, or through negotiation with nearby peers. In term of cluster size, there is a tradeoff between aggregate and individual load. It is good to choose a cluster size that is small enough to keep a reasonable individual load and provide reliability to the system, but large enough to avoid the knee in aggregate load when cluster size is small. For PALMS-SP, we employ a simple heuristic protocol for superpeer selection.

We adopt the super-peer selection protocol which is similar to the $\mathrm{H}_{2} \mathrm{O}$ (Hierarchical 2level Overlay) (Lo et al., 2005) protocol for super-peer selection. $\mathrm{H}_{2} \mathrm{O}$ is an advertisementbased super-peer selection protocol that is deployable in an unstructured overlay network. $\mathrm{H}_{2} \mathrm{O}$ uses a classic advertisement-based protocol, in which super-peers advertise superpeer information, and ordinary peers cache these advertisements. Ordinary peers can then choose to join the best super-peer using locally cached information. This protocol gives full autonomy to both super-peer and ordinary peers, allowing each to negotiate using its own local policy. $\mathrm{H}_{2} \mathrm{O}$ is similar in many ways to the gnutella protocol, but allows for finergrained control over the super-peer selection process e.g., it can consider trust, secure paths and routing performance.

The basic idea behind super-peers management mechanism for PALMS-SP is simple and intuitive. Ordinary peers with similar locality e.g., IP addresses are connected to the same super-peer. At the initial stage, all nodes start as ordinary peers. Nodes may switch role at will. The decision process is completely decentralized. An ordinary peer selects one super-peer to send queries and share resources. Since the ordinary peer depends on super-peer's capabilities, the ordinary peer should select the 
super-peer which can provide it with the best service. There are many metrics that may be used to select the best super-peer, such as average response time, bandwidth, processing capabilities, storage and so on. These metrics may have different weights depending on the objective. For PALMS-SP, we focus on response time, bandwidth and processing capabilities. In order to be selected as superpeer, ordinary peer must obtain reasonable scores for all the metrics. A super-peer can switch back to ordinary peer role only when a super-peer has lost all its clients due to nodes leaving or crashing. Super-peers exchange connected ordinary peers information at the super-layer layer. Information of connected ordinary peers is encapsulated into a UDP packet and exchanged among super-peers periodically.

\section{SCHEDULING ALGORITHMS}

The algorithms presented in this section make up the core of the PALMS-SP system. They determine how each node chooses its partner for data exchange, how data packets to be sent are chosen and scheduled, which data packets are to be requested from each connected neighbor and data to be pushed by connected super-peers.

Given the buffer maps of a node and that of its partners, a schedule is to be generated for the pull and push mechanisms for fetching the expected packets from the partners, sending packets to connected neighbors and retransmission of lost packets. Simple heuristic algorithms are used for both pull and push mechanisms.

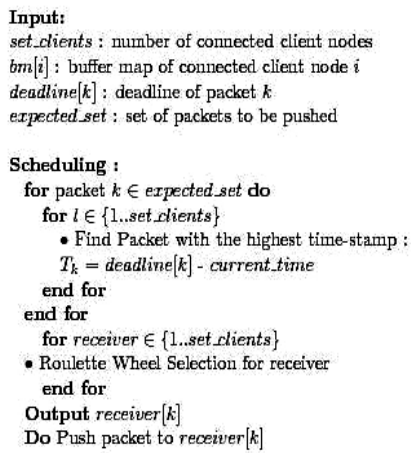

Figure 6 : Push Method Algorithm

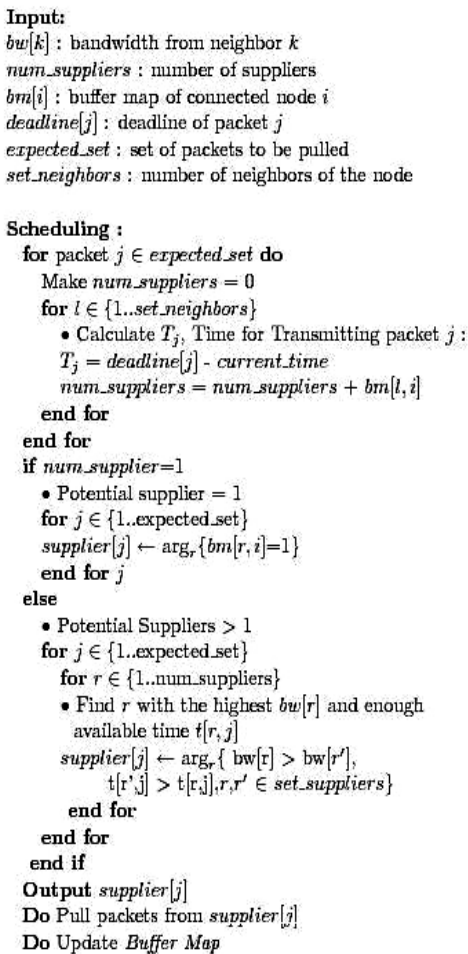

Figure 5 : Pull Method Heuristic Algorithm

The main algorithms used for peer selection for pull and push mechanisms are an altruistic algorithm.

Pull Mechanism : The algorithm for pull mechanism is similar to the heuristic used in DONet (Zhang et al., 2005) and BitTorrent (B. Cohen, 2003). The main purpose of the pull method is to request the rarest packets among those that are locally available, and to distribute the request across different possible suppliers. The pull algorithm is shown in Figure 5.

Using the information gathered from the buffer map exchanged among neighbor sets, packets that are rarest across the neighborhood are requested with higher priority than more common ones. Packets with the same number of suppliers are randomly requested from one of the neighbors that can provide them. This is to limit the load on any single peer.

Pull Mechanism : The push mechanism is the process of packet delivery by a super-peers to connected clients. Inspired by the work 
conducted by Banerjee et al. (Banerjee et al., 2006), the push mechanism for PALMS-SP employs two simple techniques too. In a nutshell, the push mechanism consists of a proactive component where data packets are pushed forward by super-peer to connected clients, and a reactive mechanism where packets are pushed forward based NACKs information received.

In order to increase delivery ratio, each superpeer at the super-peers layer, proactively send data packets to connected ordinary peers. The priority of data packets to be pushed is based on the least frequently used (LFU) policy. Due to the unreliability of the network link or a neighbor failure, some of the packets are lost during transmission. An overlay node can detect missing packet using gaps in the packet sequence numbers. This information is used to trigger NACK-based re-transmission through the next interval of push mechanism for the super-peer. Thus, with the help of the push mechanism, packets are pushed and received at the receiver nodes at a second time interval. A good selection strategy is required to distribute the packets. This is to ensure that each superpeer pushes packets that are not too close to the playout deadline and helps to reduce redundancy in push packets. Push packets also take into account the NACK requests from connected nodes. The push algorithm is shown in Figure 6.

For the push packet scheduling, in order to reduce redundancy, each super-peer tries to allocate packets that are least frequently used (LFU) into the Super-Peer Packet Map, SPPm to be pushed. A Super-Peer Packet Map, SPPm consists of node id and packet sequence number. A simple roulette wheel selection scheme is applied for the next time interval for each super-peer to push the available packets. Packets with the highest time-stamp or least sent will be given higher priority to be allocated into the Super-Peer Packet Map, SPPm. Each super-peer keeps a counter of how many times each packet is sent. Packets with the least number of times sent will be chosen. In addition to that, packets that required re-transmission based on NACKs received will be allocated into the Super-peer Packet Map, SPPm .

\section{SIMULATION SETUP}

In this section, we perform extensive simulations to study the performance of PALMS-SP. Simulations on the algorithms' behavior test for under different user arrival/departure patterns, different network sizes, bandwidth distributions, and streaming rates using network simulator ns-2 (Network Simulator).

1) Video Data: The length of the video is 120 minutes (a typical length for a movie).

2) Video Coding: We used a video stream that is MDC encoded with 5 descriptions. For simplicity, we assume that all descriptions have the same constant bit rate of $100 \mathrm{Kbps}$. Therefore, the rate of the full quality version of the stream is $500 \mathrm{Kbps}$.

3) Peer Parameters: The incoming access link bandwidths for all peers are set to $500 \mathrm{Kbps}$. The incoming access links of all peers are set to $500 \mathrm{Kbps}$ so that each peer can easily receive the full quality playback rate. The buffer length is set to 30 seconds. In all our experiments we use a heartbeat period of 5 seconds for all simulated protocols. The interval for the next round of push mechanism is set for every 5 seconds.

4) Network Topology: Topology is generated by using Georgia Tech Internetwork Topology Models (GT-ITM) generator (E. W. Zegura, 1996). The delay on the access links are randomly selected between $5 \mathrm{~ms}$ to $25 \mathrm{~ms}$. Each super-peer is connected to 10 ordinary peers in a cluster. A link between a super-peer and a normal peer is symmetric. A link between super-peers may be asymmetric because superpeers neighboring to a super-peer are randomly selected.

5) Performance Metrics: We use three basic Quality of Service (QoS) performance metrics, i.e., Average Delivery Ratio, Delivery Latency and Data Overheads. 


\section{RESULTS AND DISCUSSIONS}

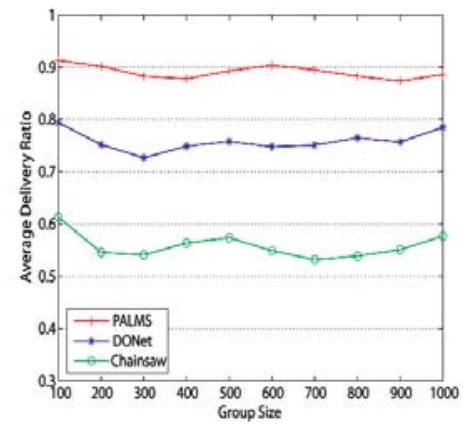

Figure 7(a) Delivery Ratio as a function to Group Size

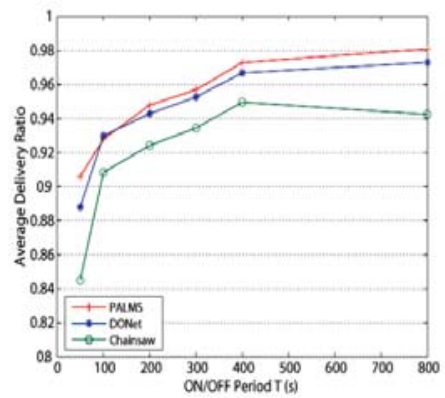

Figure 7(b) Average Delivery Ratio as a function to ON/OFF period, T(s), Group Size : 1000

We have examined the impact of heterogeneous bandwidths and different nodes arrival/departure patterns on the performance of PALMS-SP streaming. We also study the three metrics of interest: Delivery quality, Delivery latency and Data overheads. We compare the push-pull protocol performance of PALMS-SP with DONet (Zhang et al., 2005) and Chainsaw (Pai et al., 2005). Both DONet and Chainsaw employ pure pull mechanism. DONet employs a rarest-first strategy as the block scheduling method, and select suppliers with the most surplus bandwidth and enough available time first. Chainsaw uses a purely random strategy to decide what blocks to request from neighbors.

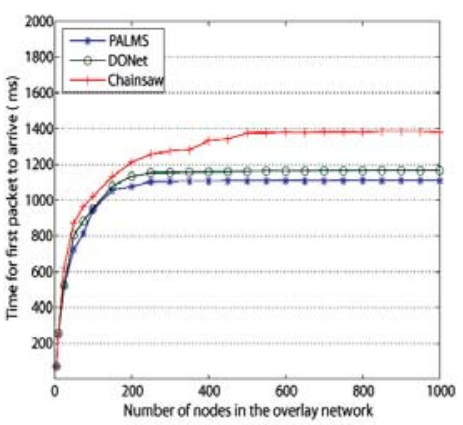

Figure 8(a) Average time for arrival of first packet

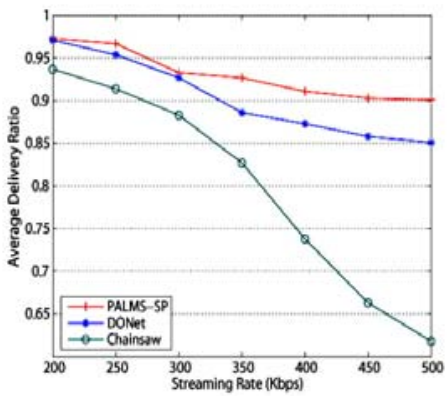

Figure 8(b) Average Delivery Ratio as a function to Streaming Rate. Group Size : 1000

Delivery Quality: Figure 7 (a) shows the average delivery ratio for PALMS-SP in comparison to DONet and Chainsaw. We define delivery ratio to represent the number of packets that arrive at each node before playback deadline over the total of number of packets encoded. We set the streaming rate as $500 \mathrm{kbps}$. From the result, we can observe that the performances for PALMS-SP and DONet remain almost the same when group size increases. This is an indication that the performance of swarming based protocols or data-driven protocols is not affected by group size. In other words, swarming protocols have a good scalability. However, Chainsaw method decreases more in comparison to PALMS-SP and DONet. As shown in Fig. 7 (a), PALMS-SP has $20 \%$ gains compared to DONet and over $45 \%$ gains compared to Chainsaw.

We also tested the performances of PALMSSP in comparison to DONet and Chainsaw under dynamic network environment. We set all the nodes to join in an initialization period of 
around 1 minute, and then we set each node changes its status according the ON/OFF model. The node actively participates the overlay during the $\mathrm{ON}$ period, and leaves (or fails) during the OFF period. Both ON and OFF periods are exponentially distributed. Figure 7(b) shows that a shorter ON/OFF period leads to a lower delivery ratio. However, the overall delivery ratio for PALMS is higher in comparison to DONet and Chainsaw because the additional push mechanism employed at the super-peer layer is able to help to recover from a vast majority of losses. Note that Chainsaw displays the poorest performance in term of delivery ratio.

Delivery Latency: In Figure 8 (a) we show the distribution of latency experienced by data packets at the different overlay nodes. In this experiment, we measure the average time for first packet arrival for all simulated protocols. Note that all protocols suffer an increase in average time of first packet arrival, stabilize, then stay relatively constant with the number of nodes. The increase is well identified and is due to the implementation of swarming protocols for PALMS-SP and DONet. As compared to DONet, the existence of super-peers and push protocol in PALMS-SP, packets have higher chances to be delivered to connected clients in a shorter period of time.

Different Streaming Rate: Figure 8 (b) shows that as the streaming rate increases, the delivery ratio for PALM-SP remains at a relatively high delivery ratio. Even when the streaming rate reaches $500 \mathrm{Kbps}$, its delivery ratio still remain above $80 \%$. This reveals that the network capacity of PALMS-SP is sufficient to support the streaming session with streaming rate of 250-500Kbps. As shown in Fig. 8 (b), PALMSSP has $3 \%$ gains of delivery ratio compared to DONet and over $13 \%$ gains compared to Chainsaw.

\begin{tabular}{|c|c|c|c|c|}
\hline \multirow{2}{*}{$\begin{array}{c}\text { Group } \\
\text { Size }\end{array}$} & \multicolumn{2}{|c|}{$\begin{array}{c}\text { Control Overheads } \\
\text { (control traffics/video } \\
\text { traffics) }\end{array}$} & \multicolumn{2}{c|}{ Average Delivery Ratio } \\
\cline { 2 - 5 } & PALMS-SP & DONet & PALMS-SP & DONet \\
\hline 100 & 0.0164 & 0.0161 & 0.91 & 0.80 \\
\hline 200 & 0.0168 & 0.0163 & 0.90 & 0.75 \\
\hline 300 & 0.0174 & 0.0171 & 0.88 & 0.72 \\
\hline 400 & 0.0181 & 0.0173 & 0.87 & 0.73 \\
\hline 500 & 0.0186 & 0.0182 & 0.89 & 0.76 \\
\hline 600 & 0.0212 & 0.0204 & 0.90 & 0.75 \\
\hline
\end{tabular}

Table 1 : Comparison of Control Overheads and Delivery Ratio for PALMS-SP and DONet with varying group sizes.

Data Overheads: In this section, we compare the overheads of PALMS-SP to DONet. Table 1 shows that PALMS-SP incurs very low additional data overheads in comparison to DONet. Control overhead is defined as the ratio of control traffic over video traffic. The control overheads at different overlay nodes increase log-arithmically with the increase in group size. The control overheads for PALMS-SP are slightly higher due to the additional messages such as Super-Peer Packet Map messages and NACKs. However the amount of increase at each overlay node is essentially minor, less than $3 \%$ of the total overall traffic. We believe the data overheads for PALMS-SP can be further reduced by increasing the window size. It can be observed that the control overhead has little relationship with the group size because each node only communicates with its neighbors and super-peer, which demonstrates the good scalability of our proposed protocol.

These results confirm the expected advantages of the proposed model PALMS-SP for P2P live media streaming.

\section{CONCLUSION}

In this paper we presented PALMS-SP, a twolayer super-peer based P2P system for live media streaming. Our system's innovative and simple features are designed with the usage of the combination push-pull protocol and the presence of two-layer super-peer based overlay network that leverages on the heterogeneity of connected nodes. 
On order to successfully deploy PALMS-SP streaming services, we proposed push-pull mechanism to address the issue of delivery quality and delivery latency. In this framework, the existence of super-peers improves delivered video quality by incorporating the proactive and the reactive push packets mechanism.

We evaluated the performance of PALMS-SP in comparison to DONet and Chainsaw. Our simulations conducted over ns-2 demonstrated that PALMS-SP delivers quite a good playback quality even under formidable network conditions i.e., heterogeneity of network bandwidths, different user arrival/departure patterns, different network sizes, and different streaming rates.

As part of our future plans, we aim to evaluate our proposed model, PALMS-SP in PlanetLab (PlanetLab), in order to further investigate the effectiveness and the robustness of our streaming model in a larger network and real network deployment. We are also keen in exploring various techniques to improve the delivered streaming quality and delivery latency.

\section{ACKNOWLEDGEMENTS}

This work was supported by the by the Ministry of Education, Culture, Sports, Science and Technology, Government of Japan, and also by a grant from the Hori Foundation Science Promotion Foundation, Japan.

\section{REFERENCES}

S. Banerjee, S. Lee, B. Bhattacharjee and A. Srinivasan, Resilient multicast using overlays, IEEE/ACM Transactions on Networking, pp. 237-248, vol. 14, no. 2, 2006.

B. Cohen, Incentives build robustness in BitTorrent, P2P Economics Workshop, Berkeley, CA, 2003.

Y. Guo, K. Suh, and D. Towsley, A Peer-toPeer on-Demand Streaming Service and Its Performance Evaluation, ICME '03: Proceedings of the 2003 International
Conference on Multimedia and Expo, pp. 649-652, Washington, DC, 2003.

M. Hefeeda, A. Habib, R. Rotev, D. Xu, and B. Bhargava, PROMISE: peer-to-peer media streaming using CollectCast, MULTIMEDIA '03: Proceedings of the eleventh ACM international conference on Multimedia, pp. 45-54, Berkeley, CA, November, 2003.

V. Lo, D. Zhou, Y. Liu, C. GauthierDickey and Jun Li, Scalable Supernode Selection in Peer-to-Peer Overlay Networks, HOT-P2P '05: Proceedings of the Second International Workshop on Hot Topics in Peer-to-Peer Systems, pp. 18-27, Washington, DC, USA, 2005.

V. N. Padmanabhan, H. J. Wang, and P. A. Chou, Resilient Peer-to-Peer Streaming, ICNP '03: Proceedings of the 11th IEEE International Conference on Network Protocols, Atlanta, GA, pp. 16-27, November, 2003.

Pai, V. Kumar, K. Tamilmani, K. Sambamurthy, V. Mohr, A. E., Chainsaw: Eliminating Trees from Overlay Multicast, IPTPS, no. 3640, pp. 127-140, 2005.

S. Ye and F. Makedon, Collaboration-aware peer-to-peer media streaming, MULTIMEDIA '04: Proceedings of the 12th annual ACM international conference on Multimedia, pp. 412-415, NY, 2004.

X. Zhang, J. Liu, B. Li, and T.P. Yum, CoolStreaming/DONet : A data-driven overlay network for efficient live media streaming, INFOCOM 2005. 24th Annual Joint Conference of the IEEE Computer and Communications Societies, vol. 3, pp. 21022111, March, 2005.

Akamai Technologies Inc, Delivery a better Internet, [Online] Available: http://www.akamai.com/.

E. W. Zegura, GT-ITM: Georgia Tech Internetwork topology models (software). http://www.cc.gatech.edu/project, 1996.

Gnutella. (2003) The annotated Gnutella protocol specification v0.4. \{Online\}. Available: http://rfcgnutella.sourceforge.net/developer/stable/ind ex.html 
Network Simulator, Ns-2.

http://www.isi.edu/nsnam/ns/

The PlanetLab project, http://www.planetlab.org/ 